Hong Kong Journal of Orthopaedic Research (An Open Access Journal for Orthopedics and Trauma Research)

\section{Research Article}

Hong Kong J Orthop Res ISSN (e): 2663-8231

ISSN (p): 2663-8223 2019; 2(3): 06-12

(C) 2019, All rights reserved www.hkorthopaedicjournal.com

\title{
Risk Factors Associated with the Development of Depression Following Sport-related Concussion
}

Jennifer I Etcheson', Nequesha S Mohamed², Iciar M Dávila Castrodad³, Wayne A Wilkie'2, Ethan A Remily², Kevin E Crutchfield ${ }^{2}$, R Frank Henn III', Ronald E Delanois ${ }^{2}$

1 Department of Orthopaedic Surgery and Rehabiliation Medicine, SUNY Downstate Medical Center, 450 Clarkson Avenue, MSC 30, Brooklyn, NY 11203

2 Department of Orthopedic Surgery, Hackensack Meridian School of Medicine at Seton Hall University, 340 Kingsland Street, Nutley, NJ 07110

3 Rubin Institute for Advanced Orthopedics, Center for Joint Preservation and Replacement, Sinai Hospital of Baltimore, 2401 West Belvedere Avenue, Baltimore, Maryland 21215

4 Department of Orthopaedic Surgery, University of Maryland School of Medicine, 22 S. Green Street, Baltimore, MD 21201

\section{Abstract}

Introduction: Repeated concussive episodes are correlated with many sequelae, including psychiatric conditions. Reports have examined the association between concussion and the development of major depressive disorder (MDD). However, no reports have evaluated patient-specific factors and post-concussion symptoms correlated with the development of MDD following a sports-related concussion (SRC). Aims and Objectives: this study examined and reported the association of patient-specific factors and post-concussion symptoms with depression following sports-related concussions. Study Design: Retrospective Review. Setting: Institutional. Materials and Methods: A retrospective review identified all patients presenting for sports-related concussion between 2012 and 2014. Patients were included if they completed a Patient Health Questionnaire (PHQ-9). Statistics: Continuous and categorical variables were assessed using Student's ttest and chi-squared test, respectively. Logistic regression analysis was conducted to assess the effect of patient-specific factors and post-concussive symptoms on the development of MDD. Results: Out of 194 patients identified (mean age= 21.2 years), 86 met criteria for a diagnosis of MDD. The MDD cohort comprised of $64.0 \%$ women and $36.0 \%$ men, whereas the without MDD consisted of $71.3 \%$ women and $28.7 \%$ men ( $p=0.276)$. Patients with MDD were significantly older than patients without MDD (24.9 vs. 17.8 years, $\mathrm{p}<0.001)$. MDD was significantly higher after SRC among patients 18 years or older compared to patients under the age of 18 ( $56.9 \mathrm{vs.} 36.9 \%, \mathrm{p}=0.007)$. There was no demonstrable difference in time to presentation between patients that developed MDD and those that did not ( 43.9 vs. 41.9 days, $p=0.864$ ). There was no difference in race for patients that developed MDD ( $p=0.748)$. With regard to sport, there were no differences found between any of the different sports played at the time of sport-related concussion $(p=0.973)$. Additionally, no significant difference was detected in cognitive disturbance $(p=0.942)$. Irritability ( 50.4 vs. $49.6 \%, p=0.012)$ and sleep disturbances $(52.2$ vs. $47.8 \%, p=0.001)$ after sustaining SRC were significantly higher among patients that developed MDD. Fatigue was not significantly different in patients with MDD after SRC $(p=0.163)$. After adjusting for confounders, logistic regression analysis demonstrated that patients $\geq 18$ years old were associated with 2.12 times higher odds of developing MDD (adjusted Odds Ratio [aOR] $=2.118, \mathrm{p}=0.023$ ) after suffering sports-related concussions. Patients with sleep disturbances after sustaining SRC had 2.57 times higher odds of developing MDD $(a O R=2.568, p=0.009)$. Patients who experienced irritability following SRC had 1.98 higher odds of developing MDD; however, this trended towards statistical significance $(p=0.054)$. Race $(p=0.893)$, sex $(p=0.601)$, sport (lacrosse: $p=0.679$; football: $p=0.327$; others: $p=0.798)$, cognitive disturbance $(p=0.884)$ and fatigue $(p=0.392)$ were not independent predictors for the development of MDD after SRC. Conclusion: Our findings indicate that patients over the age of 18 and patients who experienced sleep disturbances after sustaining a SRC have a higher likelihood of developing MDD. These findings are vital in helping providers and patients recognize the early signs and symptoms in the development of depression, while it can be promptly treated to prevent further progression.

Keywords: Sport-related Concussion, Sport Concussion, Concussion, Major Depressive Disorder, Depression.

\section{INTRODUCTION}

*Corresponding author: Ronald E. Delanois (MD) Rubin Institute for Advanced Orthopedics, Center for Joint Preservation and Replacement, Sinai Hospital of Baltimore, 2401 West Belvedere Avenue, Baltimore, Maryland 21215 Email: rdelanoi@lifebridgehealth.org
Sport-related concussions (SRCs) are a growing public health concern, with an estimated 1.6-3.8 million incidents reported annually and chronic symptoms afflicting $10-15 \%$ of individuals ${ }^{[1,2]}$. Research and media attention surrounding SRC has gained significant traction, as concussions have been correlated with many sequelae, including psychiatric conditions such as major depressive disorder (MDD) ${ }^{[3,4]}$. Post-SRC presentation is highly variable and often manifests as a combination of sleep, cognitive, psychiatric, physical, and emotional disturbances $[3,5,6]$. However, mood disturbances are among the most common symptoms experienced after SRC, with reports demonstrating up to $50 \%$ of athletes suffering from 
tendency, neuropsychological impairment $[7,8]$

The development of major depressive disorder following SRC has been shown to adversely affect the course and duration of recovery. Patients who develop MDD after SRC suffer an increase in the number of postconcussion symptoms, and at a higher level of severity ${ }^{[9]}$. Furthermore, these patients experience worse behavioral outcomes, as well as increased co-morbid cognitive impairments, anxiety disorders, substance abuse, and suicidal ideation [10-12]. In addition to acute personality changes, patients presenting with MDD after SRC have reported diminished overall quality of life and decreased functional outcome scores on par with those of advanced coronary artery disease patients ${ }^{[13,14]}$. Early identification and intervention for major depression may reduce morbidity associated with the condition and minimize patient suffering ${ }^{[15]}$.

A multitude of studies have identified strategies to decrease the incidence of SRC and to improve the welfare of individuals participating in sports. In addition, the association of SRC and the development of MDD has been examined and is well documented in the literature. However, no reports have evaluated patient-specific factors and postconcussion symptoms that are correlated with the development of MDD following a sports-related concussion. This study examines and reports the association of patient-specific factors, sport played at the time of SRC, and post-concussion symptoms with depression following sportsrelated concussions.

\section{MATERIALS AND METHODS}

\section{$\underline{\text { Database }}$}

Following Institutional Review Board (IRB) approval, a retrospective review of all patients presenting to a single traumatic brain injury (TBI) and sports medicine neurology clinic for SRC were identified between January 2012 and December 2014. The clinic is part of a larger institution and serves as a tertiary referral center for patients who are referred from the emergency department, surrounding outside hospitals, and local physicians. The database contains information such as patient demographics, date of injury/concussion, date of presentation, past and present medical history, Patient Health Questionnaire (PHQ-9) score, sport played at the time of concussion, and responses to an institutionbased neurobehavioral symptom inventory. The neurobehavioral symptom inventory assesses patients for the following post-concussion symptoms: amnesia (retrograde vs. anterograde), slurred speech, nausea or vomiting, change in appetite (increased/decreased), fatigue, numbness and tingling, headache, dizziness, irritability, cognitive disturbance (executive function, learning and memory, attention), sleep disturbance (difficulty falling or staying asleep), gait disturbance. The type of sport played at the time of concussion included the following: soccer, lacrosse, football, and an "other" category for all other sports with numbers too small to demonstrate significance. The "other" category contains cheerleading, track and field, ice hockey, and field hockey.

\section{Patient Selection}

Patients were included in the study if they had completed a PHQ-9 at the time of initial presentation to clinic and met criteria for a nonpenetrating mild traumatic brain injury, as defined by the American Congress of Rehabilitation Medicine after a sport-related injury. Patients were excluded if they had a diagnosis of MDD in their active problem list, did not complete or had missing data for the PHQ-9, and if they had a medical history significant for prior concussion, brain tumor, stroke, coronary heart disease, schizophrenia, bipolar disorder, dementia, substance abuse, or had utilized mental health services to ensure a MDD-naïve patient population.

\section{Outcome Measure}

The Patient Health Questionnaire (PHQ-9) is a tool that is utilized in primary care settings and validated for a tentative diagnosis of depression in at-risk populations (Table 1). The module scores each of the responses to the nine DSM-V criteria for depression on a scale of 0 (not at all) to 3 (nearly every day). PHQ-9 stratifies depression severity according to score: 0-4 none, 5-9 mild, 10-14 moderate, 15-19 moderately severe, $20-27$ severe. However, a score of $\geq 10$ on PHQ-9 was used to determine a diagnosis of MDD for this study, which has been validated against an independent structured mental health professional interview where a score of $\geq 10$ had a sensitivity of $88 \%$ and a specificity of $88 \%$ for MDD ${ }^{[16]}$. A score of $0-9$ was quantified as no depression based on the module's depression severity score stratification.

Table 1: Patient Health Questionnaire (PHQ-9)

\begin{tabular}{|l|l|}
\hline Score & Depression Severity \\
\hline 0 to 4 & None \\
\hline 5 to 9 & Mild \\
\hline 10 to 14 & Moderate \\
\hline 15 to 19 & Moderately Severe \\
\hline 20 to 27 & Severe \\
\hline
\end{tabular}

\section{Covariates}

A number of variables were used as covariates for statistical analysis. These included patient demographics (age, gender, race), sport played at time of SRC (soccer, football, lacrosse, other), and post-SRC symptoms (cognitive disturbance, sleep disturbance, irritability, fatigue). Age was categorized as $<18$ years old or $\geq 18$ years old. Patient race was categorized as White or as Black or African American, which were the races our patient population was comprised. Patients were divided into two groups: no MDD or MDD, as determined by the score received on the PHQ-9.

\section{Statistical Analysis}

Continuous and categorical variables were assessed using Student's ttest and chi-squared test, respectively. A binomial logistic regression model controlling for variation in patient demographics, sport played, and post-SRC symptoms (cognitive disturbance, sleep disturbance, irritability, fatigue) was conducted to assess the effect of patient-specific factors and post-concussive symptoms on the development of MDD. The threshold for statistical significance was set at a $p$-value of $<0.05$. All statistical analyses were conducted in SPSS version 25 (IBM Corporation).

\section{RESULTS}

A total of 194 patients were identified (mean age $=21.2$ years), of which 86 met criteria for a diagnosis of MDD. The cohort of patients who developed MDD comprised of $64.0 \%$ women and $36.0 \%$ men, whereas the group of patients without MDD consisted of $71.3 \%$ women and $28.7 \%$ men $(p=0.276)$ (Table 2). Patients with MDD, on average, were significantly older than patients without MDD (24.9 vs. 17.8 years, $\mathrm{p}<0.001)$. Furthermore, MDD was significantly higher after SRC among patients age 18 years or older compared to patients under the age of 18 (56.9\% vs. $36.9 \%, p=0.007)$. There was no demonstrable difference in time to presentation between patients that developed MDD and those that did not ( 43.9 vs. 41.9 days, $p=0.864$ ). There was no difference in White and Black or African American patients that developed MDD (43.9\% vs. $47.6 \%, p=0.748$ ). 
Table 2: Patient Demographics and Sport Played for Patients with and without Major Depressive Disorder

\begin{tabular}{|c|c|c|c|}
\hline & No MDD & MDD & p-value \\
\hline \multicolumn{3}{|l|}{ Sex } & \\
\hline Female & 77 & 55 & \multirow[t]{2}{*}{0.276} \\
\hline Male & 31 & 31 & \\
\hline \multicolumn{4}{|l|}{ Age } \\
\hline Mean age (years) ${ }^{*}$ & 17.8 (9 to 57$)$ & 24.9 (12 to 64$)$ & $<0.001$ \\
\hline$<18$ Years Old & 77 (63.1\%) & 45 (36.9\%) & \multirow[t]{2}{*}{0.007} \\
\hline$\geq 18$ Years Old & 31 (43.1\%) & 41 (56.9\%) & \\
\hline Time to presentation (days) & $41.9(45.6)$ & $43.9(46.2)$ & 0.864 \\
\hline \multicolumn{3}{|l|}{ Race } & \multirow[t]{3}{*}{0.748} \\
\hline Black or African American & 11 & 10 & \\
\hline White & 97 & 76 & \\
\hline \multicolumn{3}{|l|}{ Sport } & \multirow[t]{5}{*}{0.973} \\
\hline Soccer & 30 & 22 & \\
\hline Lacrosse & 25 & 20 & \\
\hline Football & 20 & 18 & \\
\hline Others & 33 & 26 & \\
\hline
\end{tabular}

MDD: Major Depressive Disorder

*The values are given as the mean and standard deviation in parentheses.

With regard to sport, there were no differences found between any of the different sports played at the time of sport-related concussion $(p=0.973)$ (Table 2). Additionally, no significant difference was detected in cognitive disturbance between patients with MDD and without (44.4\% vs. $55.6 \%, p=0.942$ ) (Table 3). Sleep disturbances after sustaining SRC were significantly higher among patients that developed MDD (52.2\% vs. $47.8 \%, p=0.001$ ). In addition, irritability following SRC was significantly higher among patients presenting with MDD $(50.4 \%$ vs. $49.6 \%, p=0.012)$. Fatigue was not found to be different in patients with MDD after SRC (46.6\% vs. $53.4 \%, p=0.163)$.

Table 3: Post-Concussion Symptoms Associated with the Development of Major Depressive Disorder

\begin{tabular}{|c|c|c|c|}
\hline Post-Concussive Symptoms & No MDD & MDD & p-value \\
\hline \multicolumn{3}{|l|}{ Cognitive Disturbance } & \multirow[t]{3}{*}{0.942} \\
\hline Yes & $90(83.3 \%)$ & 72 (83.7\%) & \\
\hline No & $18(16.7 \%)$ & $14(16.3 \%)$ & \\
\hline \multicolumn{2}{|l|}{ Sleep Disturbance } & & \multirow[t]{3}{*}{0.001} \\
\hline Yes & $64(59.3 \%)$ & $70(81.4 \%)$ & \\
\hline No & $44(40.7 \%)$ & $16(18.6 \%)$ & \\
\hline \multicolumn{3}{|l|}{ Irritability } & \multirow[t]{3}{*}{0.012} \\
\hline Yes & 66 (61.1\%) & 67 (77.9\%) & \\
\hline No & 42 (38.9\%) & 19 (22.1\%) & \\
\hline \multicolumn{3}{|l|}{ Fatigue } & \multirow[t]{3}{*}{0.163} \\
\hline Yes & $86(79.6 \%)$ & 75 (87.2\%) & \\
\hline No & $22(20.4 \%)$ & $11(12.8 \%)$ & \\
\hline
\end{tabular}

MDD: Major Depressive Disorder

After adjusting for confounders, backward logistic regression analysis demonstrated that patients $\geq 18$ years old were associated with 2.12 times higher odds of developing MDD (adjusted Odds Ratio $[a O R]=2.118, p=0.023$ ) after suffering a sports-related concussion (Table 4). Patients with sleep disturbances after sustaining SRC had 2.57 times higher odds of developing MDD ( $a O R=2.568, p=0.009$ ). Patients who experienced irritability following SRC had 1.98 higher odds of developing MDD; however, this trended towards but did not reach statistical significance $(\mathrm{aOR}=1.98, \mathrm{p}=0.054)$. Race $(\mathrm{aOR}=0.934, \mathrm{p}=0.893)$, sex (aOR=1.193, $p=0.601$ ), sport (lacrosse: $a O R=0.843, p=0.679$; football: aOR=0.650, $\quad \mathrm{p}=0.327$; others: $\mathrm{aOR}=0.890, \quad \mathrm{p}=0.798)$, cognitive disturbance $(\mathrm{aOR}=0.938, \mathrm{p}=0.884)$ and fatigue $(\mathrm{aOR}=1.450 ; \mathrm{p}=0.392)$ were not independent predictors for the development of MDD after SRC.

Table 4: Predictive Model for Developing Major Depressive Disorder Following Sports-Related Concussion

\begin{tabular}{|l|l|l|l|}
\hline Variable & Odds Ratio & $\mathbf{p}$-value & 95\% Confidence Interval \\
\hline Race & 0.934 & 0.893 & 0.345 to 2.528 \\
\hline Sex & 1.193 & 0.601 & 0.617 to 2.307 \\
\hline Age & & & \\
\hline$<18$ Years Old & ref & - & - \\
\hline$\geq 18$ Years Old & 2.118 & 0.023 & 1.107 to 4.051 \\
\hline Sport & & & \\
\hline Soccer & ref & - & - \\
\hline Lacrosse & 0.843 & 0.679 & 0.376 to 1.892 \\
\hline Football & 0.650 & 0.327 & 0.275 to 1.537 \\
\hline Others & 0.890 & 0.798 & 0.366 to 2.165 \\
\hline Post-Concussive Symptoms & & \\
\hline Cognitive Disturbance & 0.938 & 0.884 & 0.395 to 2.225 \\
\hline Sleep Disturbance & 2.568 & 0.009 & 1.265 to 5.211 \\
\hline Irritability & 1.980 & 0.054 & 0.989 to 3.965 \\
\hline Fatigue & 1.450 & 0.392 & 0.620 to 3.392 \\
\hline
\end{tabular}

\section{DISCUSSION}

Sport-related concussions have gained significant attention in the media, as emerging research has linked debilitating outcomes to the initial injury long after the trauma has occurred. Reports have focused on post-SRC symptoms including emotional and psychiatric disturbances; however, there is a paucity of literature investigating which patient groups are at increased risk for developing psychological disorders. Our study is one of the few to investigate how patient factors, sport played at time of injury, and post-concussive symptoms are associated with the development of major depressive disorder following SRC. In this analysis, patients with SRC had a greater likelihood of developing MDD if they were $\geq 18$ years old. In addition, patients that presented with sleep disturbances or irritability post-concussion demonstrated an increased likelihood of MDD.

This study is not without limitations. There may be undetectable bias, as patients who chose not to complete the Patient Health Questionnaire (PHQ-9) or had an incomplete survey were excluded from the study. However, this type of bias is inherent in all studies utilizing voluntary participation and completion of a surveys as the main resource for patient selection and analysis. Additionally, many patients do not seek treatment following concussion and we may not be capturing these patients in our results. Rather, we may be analyzing a subset of patients that are more inclined to seek medical treatment and are amenable to mental health services. However, all studies involving concussion databases will be limited to analyzing a subgroup of patients that present for treatment. Despite the above limitations, we believe this study provides important findings for physicians and healthcare providers who are interested in understanding which patients may be at higher risk for developing MDD and providing prompt treatment. 
With regard to patient age, previous studies have reported an association between age at the time of SRC and the development of MDD. Tsai et al. compared 10-24 year old patients with a diagnosis of concussion to an age- and sex-matched cohort of patients without a history of concussion to determine correlates of mood disorders, such as MDD [19]. The authors reported that compared to patients without $\mathrm{TBI}$, those with TBI were at higher risk for major depression ( $\mathrm{aHR}=2.02$, [95\% Cl 1.76-2.31]). For age-based comparisons, patients were stratified into 3 groups, with male and female patients age 10-14 years old serving as a reference. The findings indicated that males with concussion at ages 15-19 and 20-24 years had a higher risk of developing a mood disorder than those at ages 10-14 years $(\mathrm{aHR}=2.21,[95 \% \mathrm{Cl} 1.37-2.57]$ and aHR=1.33, [95\% Cl 1.03-1.73]). Similarly, females with TBI at ages 20-24 years had a higher risk than those at ages $10-14$ years (aHR=1.28, [95\% $\mathrm{Cl}$ 1.01-1.62)]. Tsai and colleagues concluded that increasing age was a factor in the occurrence of mood disorders post-concussion and may be influenced by the rising rate of psychological disorders observed in young and mature adults.

Our analysis demonstrated no difference between sport type (football, soccer, lacrosse, other) and the development of MDD following SRC. These results suggest that among the sports evaluated from our database, no sport is more predictive of developing MDD over another. Although there are limited studies comparing sport types with the development of MDD in the literature, one study demonstrated that regardless of the sport, self-reported patient mental health measures remain similar. Meehan III et al. [20] conducted a cross-sectional study of alumni athletes between ages 40 and 70 from four division III institutions who played football, men's lacrosse, ice hockey, soccer, and field hockey. The authors noted that respondents with a history of SRC had reported worse self-health regarding mood disturbances including depression, irrespective of the sport played $(p<0.001)$. In another crosssectional analysis, Gouttebarge et al. ${ }^{[21]}$ did not find an association between mental health conditions among former male professional athletes with SRC across a range of collision/contact sports (football, ice hockey, and rugby) ( $p>0.05$ ). These studies support our findings that no particular sport confers a higher predictive value for neuropsychiatric conditions such as depression.

Sleep disturbances following concussion often presents as insomnia, pleiosomnia, sleep-wake disturbances, and hypersomnia. Our study demonstrated that sleep disturbances following SRC was associated with increased odds of developing depression, which is supported by recent developments in concussion literature. A study by Raikes et al. compared white-matter properties and pathophysiology in the brains of patients with a history of concussion and patients without concussion using diffusion tensor imaging (DTI) metrics ${ }^{[22]}$. The authors found that both mean (MD) and radial diffusivity (RD) of DTI positively correlated with Pittsburgh Sleep Quality Index (PSQI) scores (corrected $p \leq 0.1$ and corrected $p<0.05$, respectively) and increasing depression on the Beck Depression Inventory-II (BDI-II) questionnaire (corrected $p<0.05$ and corrected $p<0.05$ ), while there was no correlation between selfreported outcomes and any of the DTI metrics among the healthy cohort. Furthermore, they reported that the correlations between the PSQI and BDI-II with DTI markers were observed in both hemispheres and involved white matter tracts. These tracts connect to deeper brain structures and play an integral role in sleep-wake cycles, cognitive function, executive function, attention, inhibition, and emotional control, which may be damaged following concussion. Damage to these pathways may help explain overlapping post-concussion sleep disturbances and the manifestation of psychological disorders after concussion.

\section{CONCLUSION}

Debilitating short- and long-term conditions have been linked with pathologic brain changes resulting from SRC, including mood disorders such as major depressive disorder. Our findings indicate that patients over the age of 18 have a higher likelihood of developing MDD. Additionally, patients who experienced sleep disturbances after sustaining a sport-related concussion demonstrate an increased likelihood of developing MDD. Although not statistically different, irritability following SRC was associated with an increased likelihood of MDD, which trended toward significance and may be clinically relevant. These findings demonstrate a need for early routine screening of all post-SRC patients, irrespective of age for psychiatric disorders secondary to sports-related concussion. Furthermore, it is important for physicians to have a heightened awareness of an increased risk of developing MDD in patients who present with sleep disturbances and irritability post-concussion. This, in turn, will help providers and patients recognize the early signs and symptoms in the development of depression, while it can be promptly treated to prevent further progression.

Sports-related concussions are becoming increasingly scrutinized as an increasing amount of literature is identifying long-term effects, such as worsened behavioral outcomes, and increased incidence of cognitive impairments, anxiety disorders, substance abuse, and suicidal ideation. This study empowers providers through the early identification of depression symptoms; thereby potentially avoid the long-term residual effects that result from concussions.

Conflicts of Interest: This research did not receive any specific grant from funding agencies in the public, commercial, or not-for-profit sectors.

\section{Authors' Contribution:}

Jennifer I. Etcheson, M.D., M.S.: conception of work, writing, data analysis, data interpretation

Nequesha S. Mohamed, M.D.: writing, data interpretation, editing

Iciar M. Dávila Castrodad, M.D.: writing, editing

Wayne A. Wilkie, D.O., M.H.S.A.: editing, data analysis

Ethan A. Remily, D.O.: editing, data analysis

Kevin E. Crutchfield, M.D.: acquisition of data, editing

R. Frank Henn III, M.D.: acquisition of data, editing

Ronald E. Delanois, M.D.: conception of work, acquisition of data, editing.

\section{REFERENCES}

1. Langlois JA, Rutland-Brown W, Wald MM. The epidemiology and impact of traumatic brain injury: a brief overview. J Head Trauma Rehabil n.d.; 21:375-8.

2. Daneshvar DH, Nowinski CJ, McKee AC, Cantu RC. The epidemiology of sport-related concussion. Clin Sports Med 2011; 30:1-17, vii. doi:10.1016/j.csm.2010.08.006.

3. McCrory P, Meeuwisse W, Dvořák J, Aubry M, Bailes J, Broglio S, et al. Consensus statement on concussion in sport-the 5 th international conference on concussion in sport held in Berlin, October 2016. Br J Sports Med 2017; 51:838-47. doi:10.1136/bjsports-2017-097699.

4. Jorge R, Robinson RG. Mood disorders following traumatic brain injury. NeuroRehabilitation 2002; 17:311-24.

5. Terry DP, Brassil M, Iverson GL, Panenka WJ, Silverberg ND. Effect of depression on cognition after mild traumatic brain injury in adults. Clin Neuropsychol 2018:1-13. doi:10.1080/13854046.2018.1459853. 
6. Rabinowitz AR, Levin HS. Cognitive sequelae of traumatic brain injury. Psychiatr Clin North Am 2014; 37:1-11. doi:10.1016/j.psc.2013.11.004.

7. Kontos AP, Covassin T, Elbin RJ, Parker T. Depression and neurocognitive performance after concussion among male and female high school and collegiate athletes. Arch Phys Med Rehabil 2012; 93:1751-6. doi:10.1016/j.apmr.2012.03.032.

8. Ellis MJ, Ritchie $L$, Koltek M, Hosain S, Cordingley D, Chu S, et al. Psychiatric outcomes after pediatric sports-related concussion. J Neurosurg Pediatr 2015; 16:709-18. doi:10.3171/2015.5.PEDS15220.

9. Barker-Collo S, Jones K, Theadom A, Starkey N, Dowell A, McPherson K, et al. Neuropsychological outcome and its correlates in the first year after adult mild traumatic brain injury: A population-based New Zealand study. Brain Inj 2015; 29:1604-16. doi:10.3109/02699052.2015.1075143.

10. Larson EB, Leahy B, Duff KM, Wilde MC. Assessing executive functions in traumatic brain injury: an exploratory study of the Executive Interview. Percept Mot Skills 2008; 106:725-36. doi:10.2466/pms.106.3.725-736.

11. Lewinsohn PM, Rohde P, Seeley JR. Psychosocial characteristics of adolescents with a history of suicide attempt. J Am Acad Child Adolesc Psychiatry 1993; 32:60-8. doi:10.1097/00004583-199301000-00009.

12. Moore E, Indig D, Haysom L. Traumatic brain injury, mental health, substance use, and offending among incarcerated young people. J Head Trauma Rehabil 2014; 29:239-47. doi:10.1097/HTR.0b013e31828f9876.

13. Wells KB, Stewart A, Hays RD, Burnam MA, Rogers W, Daniels M, et al. The functioning and well-being of depressed patients. Results from the Medical Outcomes Study. JAMA 1989; 262:914-9.

14. Fann JR, Katon WJ, Uomoto JM, Esselman PC. Psychiatric disorders and functional disability in outpatients with traumatic brain injuries. Am J Psychiatry 1995; 152:1493-9. doi:10.1176/ajp.152.10.1493.

15. Goldman LS, Nielsen NH, Champion HC. Awareness, diagnosis, and treatment of depression. J Gen Intern Med 1999; 14:569-80.

16. Kroenke K, Spitzer RL, Williams JB. The PHQ-9: validity of a brief depression severity measure. J Gen Intern Med 2001; 16:606-13.

17. Chrisman SPD, Richardson LP. Prevalence of diagnosed depression in adolescents with history of concussion. J Adolesc Health 2014; 54:582-6. doi:10.1016/j.jadohealth.2013.10.006.

18. Vargas G, Rabinowitz A, Meyer J, Arnett PA. Predictors and prevalence of postconcussion depression symptoms in collegiate athletes. J Athl Train 2015; 50:250-5. doi:10.4085/1062-6050-50.3.02.

19. Tsai M-C, Tsai K-J, Wang H-K, Sung P-S, Wu M-H, Hung K-W, et al. Mood disorders after traumatic brain injury in adolescents and young adults: a nationwide population-based cohort study. J Pediatr 2014; 164:136141.e1. doi:10.1016/j.jpeds.2013.08.042.

20. Meehan WP, Taylor AM, Berkner P, Sandstrom NJ, Peluso MW, Kurtz MM, et al. Division III Collision Sports Are Not Associated with Neurobehavioral Quality of Life. J Neurotrauma 2016; 33:254-9. doi:10.1089/neu.2015.3930.

21. Gouttebarge V, Aoki H, Lambert M, Stewart W, Kerkhoffs G. A history of concussions is associated with symptoms of common mental disorders in former male professional athletes across a range of sports. Phys Sportsmed 2017; 45:443-9. doi:10.1080/00913847.2017.1376572.

22. Raikes AC, Bajaj S, Dailey NS, Smith RS, Alkozei A, Satterfield BC, et al. Diffusion Tensor Imaging (DTI) Correlates of Self-Reported Sleep Quality and Depression Following Mild Traumatic Brain Injury. Front Neurol 2018; 9:468. doi:10.3389/fneur.2018.00468. 